\title{
Predictions of Diffractive, Elastic, Total, and Total-Inelastic pp Cross Sections vs LHC Measurements*
}

\section{Konstantin GOULIANOS'The Rockefeller University}

E-mail: dino@rockefeller.edu

Predictions of diffractive, elastic, total, and total-inelastic pp cross sections based on the renormalization model for diffraction are compared with recent measurements at the LHC.

XXI International Workshop on Deep-Inelastic Scattering and Related Subject-DIS2013, 22-26 April 2013

Marseilles,France

*This is an update of my contribution to the proceedings of DIFFRACTION 2012 [1] with new results on the total-inelastic and the single- and double-diffractive cross sections.

${ }^{\dagger}$ Speaker. 


\section{Introduction}

Measurements at the LHC have shown that there are sizable disagreements among Monte Carlo (MC) implementations of "soft" processes based on cross sections proposed by various physics models, and that it is not possible to reliably predict all such processes, or even all aspects of a given process, using a single model [2]. In the CDF studies of diffraction at the Tevatron, all processes are well modeled by the MBR (Minimum Bias Rockefeller) MC simulation, which is a stand-alone simulation based on a unitarized Regge-theory model, RENORM [3], employing inclusive nucleon parton distribution functions (PDF's) and QCD color factors. The RENORM model was updated in a presentation at EDS-2009 [4] to include a unique unitarization prescription for predicting the total $p p$ cross section at high energies, and that update has been included as an MBR option for simulating diffractive processes in PYTHIA8 since version PYTHIA8.165 [5], to be referred hereforth as PYTHIA8-MBR. In this paper, we briefly review the cross sections [6] implemented in this option of PYTHIA8 and compare them with LHC measurements.

The PYTHIA8-MBR option includes a full simulation of the hadronization of the implemented diffraction dissociation processes: single, double, and central diffraction. In the original MBR simulation used in CDF, the hadronization of the final state(s) was based on a data-driven phenomenological model of multiplicities and $p_{t}$ distributions calibrated using $\mathrm{S} \bar{p} p \mathrm{~S}$ and Fermilab fixed-target results. Later, the model was successfully tested against Tevatron MB and diffraction data. However, only $\pi^{ \pm}$and $\pi^{0}$ particles were produced in the final state, with multiplicities obeying a statistical model of a modified Gamma distribution that provided good fits to experimental data [7]. This model could not be used to predict specific-particle final states.

In the PYTHIA8-MBR implementation, hadronization is performed by PYTHIA8 tuned to reproduce final-state distributions in agreement with MBR's, with hadronization done in the PYTHIA8 framework. Thus, all final-state particles are now automatically produced, greatly enhancing the horizon of applicability of this simulation.

\section{Cross sections}

The following diffraction dissociation processes are considered in PYTHIA8-MBR:

$$
\begin{aligned}
& \text { SD } \quad p p \rightarrow X p \text { Single Diffraction (or Single Dissociation), } \\
& \text { or } \quad p p \rightarrow p Y \quad \text { (the other proton survives) } \\
& \text { DD } p p \rightarrow X Y \quad \text { Double Diffraction (or Double Dissociation), } \\
& \text { CD (or DPE) } p p \rightarrow p X p \text { Central Diffraction (or Double Pomeron Exchange). }
\end{aligned}
$$

The RENORM predictions are expressed as unitarized Regge-theory formulas, in which the unitarization is achieved by a renormalization scheme where the Pomeron $(\mathbb{P})$ flux is interpreted as the probability for forming a diffractive (non-exponentially suppressed) rapidity gap and thereby its integral over all phase space saturates when it reaches unity. Differential cross sections are expressed in terms of the $\mathbb{P}$-trajectory, $\alpha(t)=1+\varepsilon+\alpha^{\prime} t=1.104+0.25\left(\mathrm{GeV}^{-2}\right) \cdot t$, the $\mathbb{P}$ - $p$ coupling, $\beta(t)$, and the ratio of the triple- $\mathbb{P}$ to the $\mathbb{P}$ - $p$ couplings, $\kappa \equiv g(t) / \beta(0)$. For large rapidity 
gaps, $\Delta y \geq 3$, for which $\mathbb{P}$-exchange dominates, the cross sections may be written as,

$$
\begin{aligned}
\frac{d^{2} \sigma_{S D}}{d t d \Delta y} & =\frac{1}{N_{\text {gap }}(s)}\left[\frac{\beta^{2}(t)}{16 \pi} e^{2[\alpha(t)-1] \Delta y}\right] \cdot\left\{\kappa \beta^{2}(0)\left(\frac{s^{\prime}}{s_{0}}\right)^{\varepsilon}\right\}, \\
\frac{d^{3} \sigma_{D D}}{d t d \Delta y d y_{0}} & =\frac{1}{N_{\text {gap }}(s)}\left[\frac{\kappa \beta^{2}(0)}{16 \pi} e^{2[\alpha(t)-1] \Delta y}\right] \cdot\left\{\kappa \beta^{2}(0)\left(\frac{s^{\prime}}{s_{0}}\right)^{\varepsilon}\right\}, \\
\frac{d^{4} \sigma_{D P E}}{d t_{1} d t_{2} d \Delta y d y_{c}} & =\frac{1}{N_{\text {gap }}(s)}\left[\Pi_{i}\left[\frac{\beta^{2}\left(t_{i}\right)}{16 \pi} e^{2\left[\alpha\left(t_{i}\right)-1\right] \Delta y_{i}}\right]\right] \cdot \kappa\left\{\kappa \beta^{2}(0)\left(\frac{s^{\prime}}{s_{0}}\right)^{\varepsilon}\right\},
\end{aligned}
$$

where $t$ is the 4-momentum-transfer squared at the proton vertex, $\Delta y$ the rapidity-gap width, and $y_{0}$ the center of the rapidity gap. In Eq. (2.6), the subscript $i=1,2$ enumerates Pomerons in the DPE event, $\Delta y=\Delta y_{1}+\Delta y_{2}$ is the total rapidity gap (sum of two gaps) in the event, and $y_{c}$ is the center in $\eta$ of the centrally-produced hadronic system.

The total cross section $\left(\sigma_{\text {tot }}\right)$ is expressed as:

$$
\begin{array}{ll}
\sigma_{\mathrm{tot}}^{p^{ \pm} p}=16.79 s^{0.104}+60.81 s^{-0.32} \mp 31.68 s^{-0.54} & \text { for } \sqrt{s} \leq 1.8 \mathrm{TeV}, \\
\sigma_{\mathrm{tot}}^{p^{ \pm} p}=\sigma_{\mathrm{tot}}^{\mathrm{CDF}}+\frac{\pi}{s_{0}}\left[\left(\ln \frac{s}{s_{F}}\right)^{2}-\left(\ln \frac{s^{\mathrm{CDF}}}{s_{F}}\right)^{2}\right] & \text { for } \sqrt{s} \geq 1.8 \mathrm{TeV},
\end{array}
$$

where $s_{0}$ and $s_{F}$ are energy and (Pomeron flux) saturation scales, respectively [6]. For $\sqrt{s} \leq 1.8$ $\mathrm{TeV}$, where there are Reggeon contributions, we use the global fit expression [8], while for $\sqrt{s} \geq 1.8$ $\mathrm{TeV}$, where Reggeon contributions are negligible, we employ the Froissart-Martin formula [9, 10, 11]. The two expressions are smoothly matched at $\sqrt{s} \geq 1.8 \mathrm{TeV}$.

The elastic cross section is obtained from the global fit [8] for $\sqrt{s} \leq 1.8 \mathrm{TeV}$, while for $1.8<$ $\sqrt{s} \leq 50 \mathrm{TeV}$ we use an extrapolation of the global-fit ratio of $\sigma_{\mathrm{el}} / \sigma_{\mathrm{tot}}$, which is slowly varying with $\sqrt{s}$, multiplied by $\sigma_{t o t}$. The total non-diffractive cross section is then calculated as $\sigma_{\mathrm{ND}}=$ $\left(\sigma_{\mathrm{tot}}-\sigma_{\mathrm{el}}\right)-\left(2 \sigma_{\mathrm{SD}}+\sigma_{\mathrm{DD}}+\sigma_{\mathrm{CD}}\right)$.

\section{Results}

In this section, we present as examples of the predictive power of the RENORM model some results reported by the TOTEM, CMS, and ALICE collaborations for $p p$ collisions at $7 \mathrm{TeV}$, which can be directly compared with RENORM formulas without using the PYTHIA8-MBR simulation. Figure 1 (left) shows a comparison of the TOTEM total, elastic, and total-inelastic cross sections, along with results from other experiments, fitted by the COMPETE collaboration [12]; the RENORM predictions, displayed as filled (green) squares, are in excellent agreement with the TOTEM results. Similarly, in Fig. 1 (right), good agreement is observed between the ALICE [13] and CMS [14] total-inelastic cross sections at $\sqrt{s}=7 \mathrm{TeV}$ and the RENORM prediction.

Another example of the predictive power of RENORM is shown in Fig. 2, which displays the total SD (left) and DD (right) cross sections for $\xi<0.05$, after extrapolation into the low mass region from the measured CMS cross sections at higher mass regions(see [15]) using RENORM. 

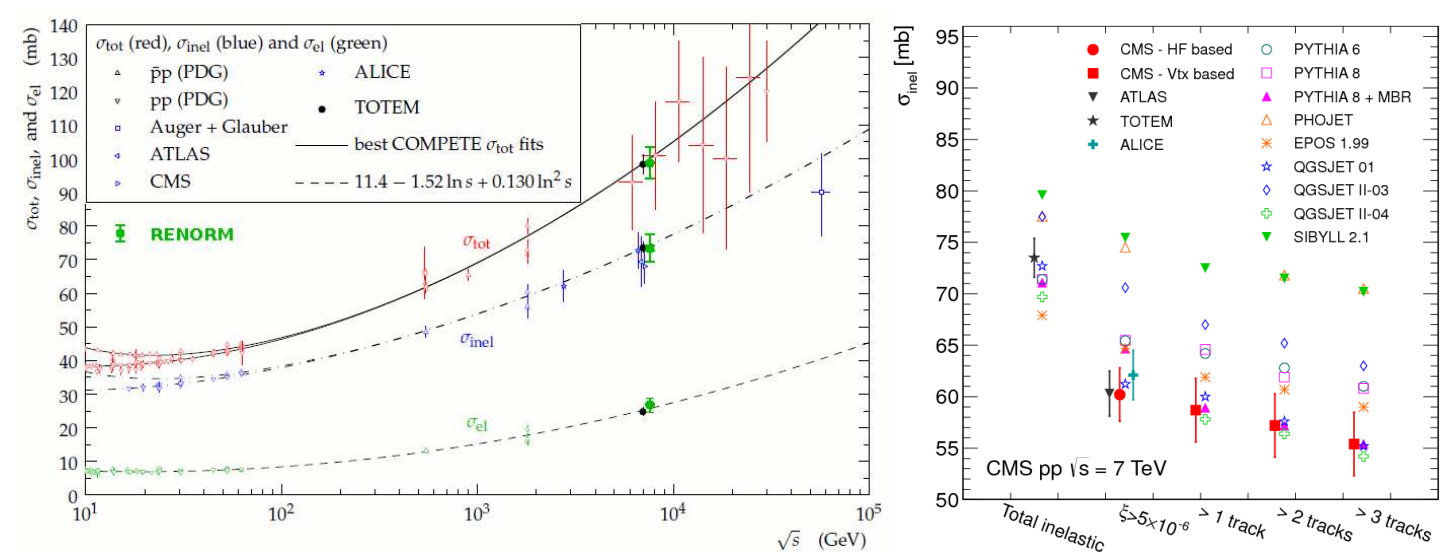

Figure 1: (left) TOTEM measurements of the total, total-inelastic, and elastic $p p$ cross sections at $\sqrt{s}=7 \mathrm{TeV}$ shown with best COMPETE fits [12], with RENORM predictions added as filled squares; (right) ALICE [13] and CMS [14] measurements of the total inelastic cross section at $\sqrt{s}=7 \mathrm{TeV}$ show good agreement with the RENORM prediction (PYTHIA8-MBR).
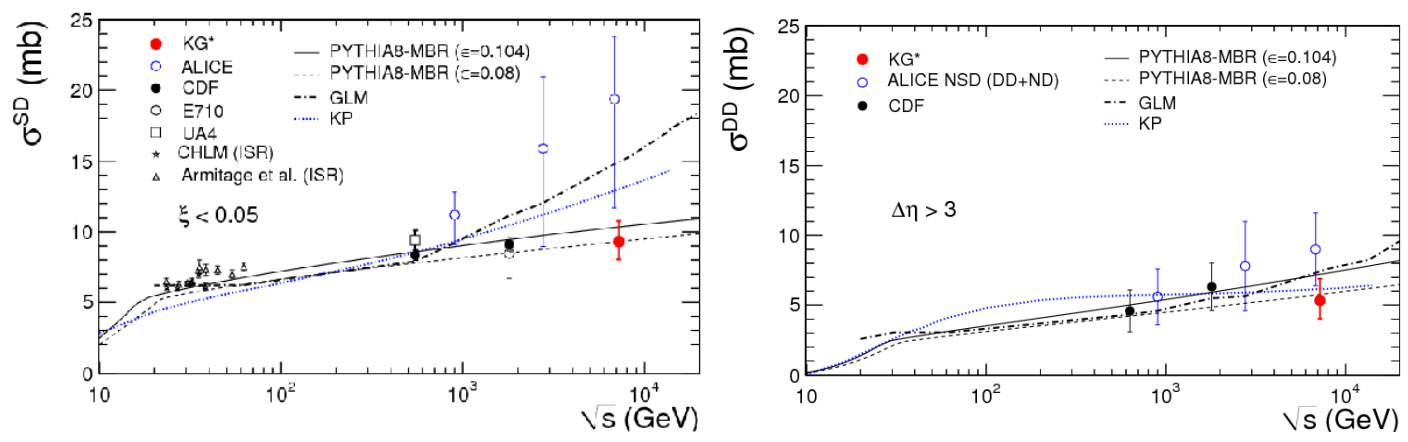

$\mathrm{KG}^{*}$ : this "data" point was obtained after extrapolation into the unmeasured low mass region(s) from the measured CMS cross sections [15] using the MBR model.

Figure 2: Measured SD (left) and DD (right) cross sections for $\xi<0.05$ compared with theoretical predictions; the model embedded in PYTHIA8-MBR provides a good description of all data.

\section{Summary}

We review our pre-LHC predictions for the total, elastic, total-inelastic, and diffractive components of the proton-proton cross section at high energies based on a special parton-model approach to diffraction employing inclusive proton parton distribution functions and QCD color factors. We discuss single diffraction, double diffraction and central diffraction or double-Pomeron exchange, and compared predictions with LHC measurements, finding good agreement with all data.

\section{Acknowledgments}

I would like to thank Robert Ciesielski, my colleage at Rockefeller and collaborator in the implementation of the MBR simulation into PYTHIA8, and the Office of Science of the Department of Energy for supporting the Rockefeller experimental diffraction physics programs at Fermilab and LHC on which this research is encored. 


\section{References}

[1] Goulianos, Konstantin, Predictions of Diffractive Cross Sections in Proton-Proton Collisions, in proceedings of Diffraction 2012: International Workshop on Diffraction in High Energy Physics, 10-15 September 2012, AIP Conf. Proc. 1523, pp. 107-110 (2013) [doi:http://dx.doi.org/10.1063/1.4802128].

[2] See models presented by various authors in proceedings of Diffraction 2012, AIP Conf. Proc. 1523.

[3] K. Goulianos, Hadronic Diffraction: Where do we Stand?, in proceedings of Les Rencontres de Physique de la Vallee d'Aoste: Results and Perspectives in Particle Physics, La Thuile, Italy, February 27 - March 6, 2004, Frascati Physics Series, Special 34 Issue, edited by Mario Greco [arXiv:hep-ph/0407035].

[4] K. Goulianos, Diffractive and Total pp Cross Sections at LHC, in proceedings of 13th International Conference on Elastic and Diffractive Scattering (Blois Workshop) - Moving Forward into the LHC Era [arXiv:1002.3527v2].

[5] T. Sjöstrand, S. Mrenna and P. Skands, JHEP05 (2006) 026, Comput. Phys. Comm. 178 (2008) 852 [arXiv:hep-ph/0603175, arXiv:0710.3820].

[6] R. Ciesielski and K. Goulianos, MBR Monte Carlo Simulation in PYTHIA8 [arXiv:1205.1446].

[7] K. Goulianos, Phys. Lett. B 193, 151 (1987).

[8] R. J. M. Covolan, J. Montanha and K. Goulianos, Phys. Lett. B 389, 176 (1996).

[9] M. Froissart, Phys. Rev. 3, 123 (1961).

[10] A. Martin, Nuovo Cimento 42, 930 (1966).

[11] A. Martin, Phys. Rev. D 80, 065013 (2009).

[12] M. Deile (тотем Collaboration), Measurements of Proton-Proton and Total Cross Section at the LHC by TOTEM, in proceedings of DIFFRACTION 2012 (figure from talk presented at the Conference).

[13] B. Abelev et al. (ALICE Collaboration), Measurement of Inelastic, Single- and Double-Diffraction Cross Sections in Proton-Proton Collisions at the LHC with ALICE, Eur. Phys. J. C (2013)73:2456, [arXiv:1208.4968], [DOI 10.1140/cpjc/s10052-013-2456-0]

[14] S. Chatrchyan et al. (CMS Collaboration), Measurement of the inelastic proto-proton cross section at $\sqrt{s}=7 \mathrm{TeV}$, Phys. Lett. B 722, 5 (2013).

[15] R. Ciesielski, Measurements of diffraction in p-p collisions in CMS, talk at this conference. 